\title{
Arteritis de Takayasu
}

\author{
Takayasu's arteritis
}

\author{
Harold Miranda, Jorge luis Blanco, Fabián Moreno • Pereira (Colombia)
}

\begin{abstract}
Resumen
Presentamos el caso de una paciente de 28 años quien consulta a nuestra clínica por pérdida bilateral y crónica de la visión y episodios de síncope de reciente aparición. No tenía historia de síntomas sistémicos o debidos a disminución del flujo sanguíneo en las extremidades. Durante la hospitalización se encuentra déficit de pulso en las extremidades superiores. La arteriografía muestra estenosis severa de ambas arterias subclavias, carótidas comunes y la arteria vertebral derecha. Se hizo un diagnóstico de arteritis de Takayasu. Se presenta una revisión corta de la patología (Acta Med Colomb 2011; 36: 93-97).
\end{abstract}

Palabras clave: Takayasu, arteritis de Takayasu, retinopatía por Takayasu.

\begin{abstract}
We describe the case of a 28 -year-old woman who presented to our clinic with a history of chronic bilateral visual loss and recent episodes of syncope. She did not have a history of systemic symptoms or manifestations of reduced blood flow in her limbs. During her hospital stay, deficient pulses were discovered in both arms. Once hospitalized the physician discloses a pulse deficit in both arms. Angiographic studies showed severe bilateral stenosis of the subclavian and carotid arteries and of the right vertebral artery. The diagnosis of Takayasu's arteritis was confirmed. A brief review of this disease is presented in this paper (Acta Med Colomb 2011; 36: 93-97).
\end{abstract}

Key-words: Takayasu, Takayasu's arteritis, Takayasu retinopathy.
Dr. Harold Miranda Rosero: Médico Internista, Profesor de Posgrado Programa de Medicina Interna, Facultad de Medicina, Universidad Tecnológica de Pereira; Dr. Jorge Luis Blanco Mosquera. Residente II de Medicina Interna; Dr. Fabián Moreno Murillo: Médico General. Universidad Tecnológica de Pereira. Pereira (Colombia). Correspondencia. Dr. Harold Miranda E-mail: hamirandaro@gmail.com Recibido: 10/XI/2010 Aceptado: 14/IV/2011

\section{Caso}

Paciente de 28 años, de ocupación secretaria, casada, G 2P2A0, procedente de área urbana de la cuidad de Dosquebradas, departamento del Risaralda. Ingresa a urgencias desde el servicio de angiografía por presentar episodio de síncope relacionado con la administración del anestésico tópico ocular para la realización de angiografía ocular bilateral sugerida por oftalmólogo retinólogo. La paciente había presentado dos episodios de pérdida del tono postural de menos de 1 min de duración en la semana previa a la consulta interpretados como síncope, el primero mientras caminaba y el segundo estando en reposo. En ninguno de los episodios reportó pródromos, relajación de esfínteres o movimientos anormales y tampoco presentó mordedura de lengua, posterior al evento, presentó recuperación rápida, espontánea y completa de sus funciones. En la revisión por sistemas la paciente presenta pérdida bilateral y progresiva de la visión de un año de evolución, al ser valorada por oftalmología y retinología consideran una probable neurorretinitis, para lo cual se solicitó la angiografía ocular. Dentro de los antecedentes la paciente no refería historia de enfermedad médica o quirúrgica previa, se había realizado cirugía de esterilización tres años antes, no había historia de exposición a tóxicos, alergias o consumo de sustancias.

Al examen físico de ingreso se encuentra una paciente en buenas condiciones generales, con presión arterial (PA) 100/60 mmHg, frecuencia cardiaca (FC) 77 por minuto, frecuencia respiratoria (FC) 12 por minuto, saturación de $\mathrm{O}_{2}$ $\left(\mathrm{Sat}_{2}\right)$ 95\% sin oxígeno suplementario. Cabeza y cuello sin alteraciones evidentes, cardiopulmonar sin alteraciones, abdomen blando, depresible, no doloroso, no se palpan masas; genitourinario sin alteración; diuresis en adecuada cantidad. Al examen neurológico se aprecian pupilas dilatadas simétricas reactivas a la luz y disminución bilateral marcada de la agudeza visual, no déficit de pares craneales, déficit focal motor ni sensitivo, reflejos $++/++++$, no reflejos patológicos. No lesiones en piel. No se reportó fondo de ojo (Tabla 1).

La paciente es hospitalizada y valorada por neurología clínica que ordena estudios de resonancia magnética cerebral con gadolinio, anticuerpos antinucleares (ANA) y solicita valoración por oftalmología.

Al tercer día de hospitalización presenta varios episodios de ortostatismo requiriendo asistencia para deambular pero sin presentar focalización motora. En un episodio de ortostatismo se encuentra que la presión arterial no se detectaba 
Tabla 1. Exámenes de laboratorio de ingreso.

\begin{tabular}{|l|l|}
\hline Leucocitos $13860 \mathrm{x} \mathrm{mm} \mathrm{mm}^{3}$ & Neutrófilos $60.5 \%$ \\
Monocitos $10.4 \%$ & Linfocitos $27.5 \%$ \\
Hemoglobina $10.02 \mathrm{~g} / \mathrm{dL}$ & Hematocrito $31.5 \%$ \\
VCM $72.1 \mathrm{fL}$ & RDW $16.3 \%$ \\
Plaquetas $663.100 \mathrm{x} \mathrm{mm}{ }^{3}$ & Tiempo de protrombina $13.5 \mathrm{seg}$. INR 1.3 \\
Tiempo Parcial de tromboplastina (TPT) $38.1 \mathrm{seg}$ & Velocidad de sedimentación globular (VSG): $60 \mathrm{~mm} / \mathrm{h}$ \\
Proteína C reactiva (PCR): $130.5 \mathrm{mg} / \mathrm{L}$ & Alanino aminotransferasa (ALT) $24 \mathrm{U} / \mathrm{L}$ \\
Aspartato aminotransferasa (AST) $18 \mathrm{U} / \mathrm{L}$ & Serología para sífilis (VDRL): negativa \\
ELISA para HIV: negativo & ANAS negativos \\
Tomografía cerebral simple normal & Radiografía de tórax normal \\
\hline
\end{tabular}

con tensiómetro manual y electrónico, con los siguientes signos vitales: FC: $126 / \mathrm{min}, \mathrm{FR}: 18 / \mathrm{min}$ y $\mathrm{SatO}_{2}: 96 \%$ con $\mathrm{FIO}_{2}: 21 \%$. Al examen físico se encuentra que no hay déficit neurológico focal, signos de bajo gasto cardiaco, fiebre, compromiso respiratorio o de otro sistema. Luego de un periodo de pocos minutos en reposo se reevaluó y se verificó que la presión arterial no era detectable en las extremidades superiores, en extremidades inferiores se encontró en 160/80 $\mathrm{mmHg}$. Los pulsos radiales, humerales y carotídeos estaban disminuidos mientras que los pulsos pedios, poplíteos e inguinales estaban presentes de buena intensidad. Con estos hallazgos se solicitó un doppler arterial de extremidades superiores, carótidas, vasos abdominales y pélvicos.

El reporte del doppler arterial de vasos cervicales fue el siguiente: "marcada hiperplasia de la íntima a nivel de las arterias carótidas comunes y disminución de la luz vascular de $75 \%$ de manera bilateral".

El doppler de extremidades superiores reportó: marcada disminución del flujo, lo que dificulta la visualización de las arterias subclavias de manera bilateral, con escaso flujo en la parte distal de las arterias radial y ulnar del brazo izquierdo.

El doppler de los vasos abdominales y pélvico se reportó dentro de parámetros normales.

En dicho contexto se realizó una arteriografía de aorta y extremidades superiores que reportó una estenosis de la mayor parte de las arterias carótidas comunes y vertebral derecha, oclusión de ambas arterias subclavias, cayado aórtico y segmento possubclavio de calibre normal, hallazgos que eran compatibles con arteritis de Takayasu (Figuras 1 y 2).

Fue valorada por retinología que indicó que los hallazgos al fondo de ojo eran compatibles con enfermedad de Takayasu.

Se programó para intervención endovascular de las estenosis arteriales documentadas.

\section{Discusión}

Presentamos el caso de una mujer joven que se presenta con un cuadro de disminución crónica de la agudeza visual, episodios de pérdida transitoria de tono postural de reciente

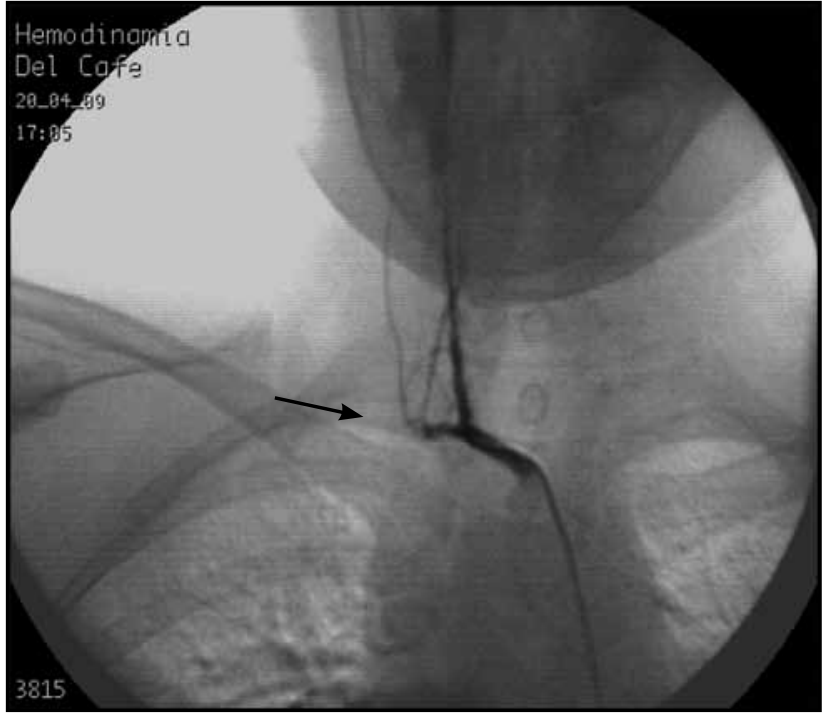

Figura 1. Arteriografía con compromiso de la arteria subclavia izquierda.

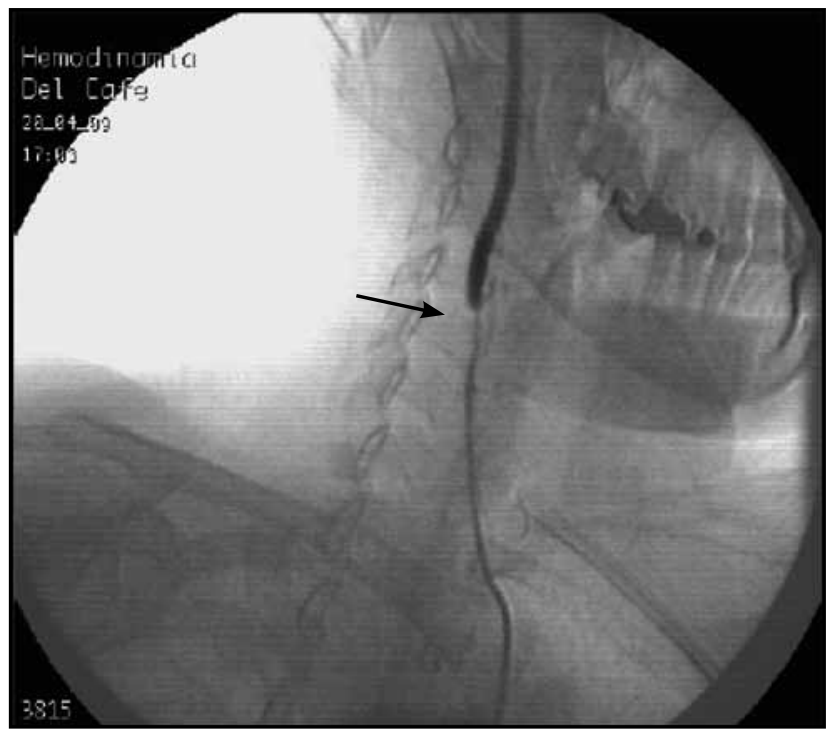

Figura 2. Arteriografía con compromiso de carótida derecha. 
aparición, asociados a elevación de la VSG y PCR, y una anemia microcítica hipocrómica.

Con respecto a los episodios de pérdida del tono postural se debe tener en cuenta dentro del diagnóstico diferencial episodios convulsivos, trastornos causantes de síncope, trastornos del movimiento como mioclonías o coreo atetosis, trastornos psicológicos como hiperventilación o crisis de pánico, hipoglucemia, consumo de medicamentos como antidepresivos tricíclicos, alfabloqueadores, pseudoefedrina, cocaína o metanfetaminas y un accidente isquémico transitorio.

No hay elementos para considerar un cuadro convulsivo entre otros por la duración de los episodios, la ausencia de aura, movimientos tonicoclónicos, relajación de esfínteres o episodio posictal prolongado. La paciente no tiene historia de enfermedad psiquiátrica o consumo de sustancias psicoactivas. No presenta el miedo y angustia de aparición súbita característicos de una crisis de pánico. La recuperación de la conciencia en los tres episodios fue espontánea y no se documentó hipoglucemia.

Si nos ceñimos a la definición del TIA Working Group que consideran el AIT como un episodio breve de disfunción focal originado por isquemia cerebral o retiniana, cuyos síntomas suelen durar menos de una hora y sin evidencia de infarto cerebral (17) sería una de las condiciones clínicas por considerar.

En cuando a la pérdida bilateral crónica de la visión que presenta esta paciente se deben considerar las lesiones compresivas del quiasma óptico, degeneración macular relacionada con la edad, toxicidad crónica por medicamentos como cloroquina o etambutol, sarcoidosis, vasculitis y colagenosis como causa (1). No hay datos de alteración de los campos visuales como hemianopsia o cuadrantopsia para pensar en compromiso del quiasma óptico y no hay referencia de cataratas por retinología. La degeneración macular relacionada con la edad es una patología de pacientes mayores de 50 años (2). La paciente no tiene historia de consumo de cloroquina o etambutol. No hay datos clínicos que soporten una sarcoidosis como el compromiso pulmonar, no hay lesiones cutáneas, manifestaciones cardiacas, ni adenopatías.

La paciente no tiene historia de miopatía, artritis, fenómeno de Reynaud, xerosis o lesiones cutáneas para considerar enfermedades del tejido conectivo.

Cuando hay desproporción entre la importancia de los síntomas generales y la escasez de hallazgos clínicos y/o manifestaciones del sistema nerviosos central en una paciente jóven cabe la posibilidad de una vasculitis.

Sin compromiso cutáneo, pulmonar o renal, cabe la posibilidad de una vasculitis de grandes vasos. Se descartó compromiso luético.

Para esta paciente la arteritis de Takayasu y la arteritis de células gigantes, son las vasculitis que afectan más comúnmente el arco aórtico. Estas dos comparten etiología, autoinmunidad mediada por células, tamaño de los vasos afectados, síntomas isquémicos relacionados con la hiper- plasia de la íntima de los vasos afectados y la respuesta a corticoides $(3,4)$.

Sin embargo, la arteritis de células gigantes afecta a personas mayores de 50 años, y se caracteriza por cefalea, sensibilidad a la palpación de la arteria temporal, VSG mayor de $50 \mathrm{~mm} / \mathrm{h}$, la pérdida de la agudeza visual puede ser aguda o subaguda y la amaurosis fugax es una manifestación común (5).

En una mujer joven alteración neurológica, reactantes de fase aguda elevados, pulsos arteriales alterados o frémitos sobre grandes arterias nos haría pensar en arteritis de Takayasu (2).

Dadas las caracteristicas de la paciente y los hallazgos angiográficos se hace diagnóstico de arteritis de Takayasu.

\section{Arteritis de Takayasu}

La arteritis de Takayasu es una vasculitis crónica de etiología no conocida, las mujeres son afectadas en 80 a $90 \%$ de los casos, con edad de inicio usualmente entre los 10 y 40 años. Tiene amplia distribución mundial, con gran prevalencia en Asia (6).

Esta enfermedad fue descrita por primera vez en 1908 por el oftalmólogo japonés Mikito Takayasu, quien reportó el caso de una mujer de 21 años con pérdida súbita de la visión y anastomosis arteriovenosas alrededor del disco óptico. Sin embargo, hay descripciones previas como la de Morgagni en 1761 de una mujer de 40 años con múltiples estenosis y aneurismas en los grandes vasos, en quien los médicos habían notado la ausencia de pulsos radiales en la misma paciente años antes de su muerte, o la del Dr. R. Rokushu Yamamoto en 1830 (7).

El estudio de Cañas y col. muestra que en Colombia la edad de inicio está entre los 13 y 47 años y que la edad de los pacientes al momento del diagnóstico fue $30.6+/-9.4$ años, se encontró igualmente un predominio de la enfermedad en mujeres (relación 2.9:1) (8).

\section{Cuadro clínico}

La enfermedad tiene dos fases distinguibles, una fase aguda y una fase crónica.

En la fase aguda el paciente presenta síntomas generales que se relacionan con la severidad de la inflamación vascular como fiebre prolongada, malestar general, pérdida de peso, mialgias, elevación de reactantes de fase aguda, trombocitosis que representan el efecto de la citoquinas, particularmente interleuquina 6 producida en las áreas de inflamación aguda $(9,10)$. A medida que progresa la inflamación vascular se presentan cambios irreversibles de la pared vascular como estenosis en la mayoría de los casos, aneurismas o cambios mixtos; estos cambios se manifiestan como síntomas explicables por insuficiencia vascular como claudicación de las extremidades, soplos carotídeos o disminución de los pulsos en las extremidades, particularmente las superiores con la afectación de las arterias subclavias, tronco braquiocefálico, femorales 
o iliacas. El compromiso de las arterias renales resulta en hipertensión arterial. Los síntomas se relacionan con el territorio vascular afectado (11).

En la arteritis de Takayasu los síntomas neurológicos se presentan en más de la mitad de los pacientes y se producen como consecuencia de la estenosis de las arterias carótidas y/o vertebrales. Las manifestaciones más frecuentes son cefalea, vértigo, inestabilidad, síntomas ortostáticos y síncope. Otras complicaciones más graves, como accidente cerebrovascular isquémicos ocurren en 5\% de los pacientes. Las alteraciones visuales, como la amaurosis fugaz o pérdida de visión permanente se producen en $10 \%$ de los casos. Otra causa importante de disminución o pérdida de la agudeza visual, sobre todo en pacientes de raza asiática, es la retinopatía hipotensiva, producida por la hipoperfusión de los vasos retinianos y coroidales, secundaria a la estenosis de las arterias carótidas (12).

\section{Patología}

Inflamación activa es manifestada por la presencia de células mononucleares, especialmente histiocitos, linfocitos y macrófagos. Con la destrucción de la capa media muscular y la lámina elástica se pueden producir aneurismas. Con la progresión del proceso inflamatorio, la proliferación de la íntima contribuye al desarrollo de lesiones estenóticas. $\mathrm{La}$ arteritis de Takayasu se caracteriza por una panarteritis que inicia en la adventicia y progresa hacia la íntima (11).

\section{Diagnóstico}

El diagnóstico de esta entidad se hace con base en los criterios del Colegio Americano de Reumatología publicados en 1990. Los criterios diagnósticos propuestos por la Asociación Americana de Reumatología (ARA) en 1990 son: (6)
- Edad de inicio menor o igual a 40 años.

- Claudicación de extremidades.

- Disminución de pulso en una o ambas arterias braquiales.

- Diferencia de al menos $10 \mathrm{mmHg}$ en la presión arterial sistólica entre ambas extremidades.

- Soplo audible en subclavias o aorta.

- Anormalidades en la arteriografía.

Nuestra paciente cumple con tres criterios para diagnóstico de arteritis de Takayasu.

Se dice que un paciente tiene arteritis de Takayasu, si tiene al menos tres de los seis criterios descritos (sensibilidad $90.5 \%$ y especificidad $97.8 \%$ ).

Desde el punto de vista imagenológico se puede clasificar según el territorio afectado así: (Figura 3) en nuestro caso la angiografía de la paciente es compatible con tipo I de la arteritis de Takayasu (13).

\section{Hallazgos de laboratorios}

Los hallazgos de laboratorios reflejan el proceso inflamatorio, pero no son específicos. En la mayoría de los pacientes se encuentra anemia de enfermedad crónica. Los reactantes de fase aguda como velocidad de sedimentación eritrocitaria, incremento de la proteína $\mathrm{C}$ reactiva, autoanticuerpos asociados a otras formas de enfermedad vascular, incluyendo anticuerpos antinucleares, anticuerpos anticitoplasma de neutrófilos y anti-DNA y anticuerpos antifosfolípidos no son encontrados en arteritis de Takayasu (14).

\section{Estudios de imágenes}

Una amplia variedad de anormalidades con diferentes estudios de imágenes pueden ser observadas en pacientes

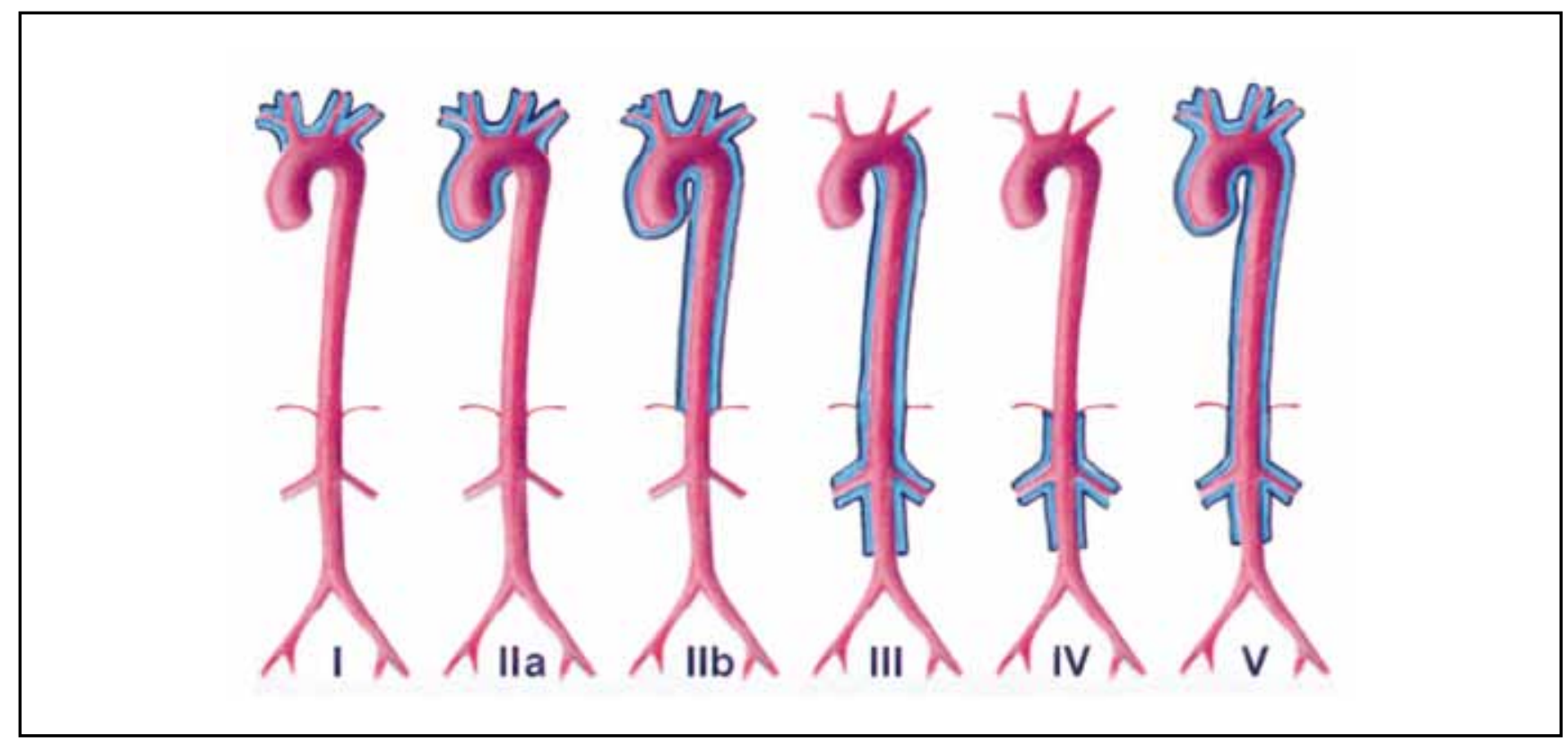

Figura 3. Tomado de RadioGraphics 2004; 24:773-786. 
con arteritis de Takayasu. En muchos casos el diagnóstico es basado en los hallazgos de imágenes del árbol arterial realizado por angioresonancia, CT scan y angiografía convencional.

La angiografía convencional ha sido utilizada tradicionalmente para el diagnóstico de la arteritis de Takayasu. Algunas técnicas no invasivas como la ecografía, la tomografía y la resonancia magnética también han sido utilizadas. El ultrasonido permite detectar cambios en el grosor de la pared del vaso, localización, longitud y severidad de las lesiones, además, determinar si la obstrucción vascular es secundaria a placas ateromatosas. La tomografía permite evaluar cambios en la pared y estenosis de la aorta y sus grandes vasos; sin embargo, esta técnica posee menores detalles de resolución que el ultrasonido y conlleva el riesgo inherente a los medios de contraste. Existen nuevas modalidades de imágenes que ayudan a hacer el diagnóstico de manera precoz y precisa, tales como la angiorresonancia y la tomografía por emisión de positrones $(15,16)$.

En este caso se utilizó angiografía como criterio diagnóstico que muestra severo compromiso tipo I (Figuras 1 y 2).

\section{Conclusión}

La enfermedad de Takayasu es una entidad relativamente infrecuente en nuestro medio y se debe tener un alto índice de sospecha para su diagnóstico y tratamiento. Es de anotar la importancia que tiene la historia clínica y el examen físico completo para llegar a un diagnóstico acertado y evitar dilaciones que derivan en demoras en el inicio de un adecuado tratamiento.

\section{Referencias}

1. Vortmann M, Schneider J. Acute monocular visual loss. Emerg Med Clin N Am 2008; 26: 73-96.

2. Jager R, Mieler W, Miller J. Age related macular degeneration. $N$ Engl J Med 2008; 358: 2606-17.

3. Arnaud L, Kahn JE, Girszyn N, Piette AM, Bletry O, et al. Takayasu's arteritis: Au update on physiopathology. Eur J Intern Med 2006; 17: 241-246

4. Michel, BA, Arend, WP, Hunder, GG. Clinical differentiation between giant cell (temporal) arteritis and Takayasu's arteritis. J Rheumatol 1996; 23: 106

5. Minagar A, Fowler, M, Harris M, Jaffe S. Neurologic Presentations of Systemic Vasculitides. Neurol Clin N Am 2010; 28: 171-184.

6. Arend WP, Michel BA, Bloch DA, Hunder GG, Calabrese LH, Edworthy SM, et al. The American College of Rheumatology 1990 criteria for the classification of Takayasu arteritis. Arthritis Rheum 1990; 33:1129.

7. Numano F, Okawara M, Inomata H.. Takayasu's arteritis. Lancet 2000; 356: 1023-5.

8. Cañas CA, Jimenez CA, Ramirez LA, Uribe O, Tobón I, Torrenegra A, et al. Takayasu in Colombia. Int J Cardiol 1998; 66: 73-9.

9. Noris M, Daina E, Gamba S, Bonazzola S, Remuzzi G. Interleukin-6 and RANTES in Takayasu arteritis: a guide for therapeutic decisions?. Circulation 1999; 100: 55 .

10. Stone J. Vasculitis: A Collection of Pearls and Myths. Rheum Dis Clin N Am 2007; 33: 691-739.

11. Johnston SL, Lock RJ, Gompels MM. Takayasu arteritis: a review. J Clin Patho 2002; 55: 481-6.

12.Hernández-Rodríguez J, Espinosa G, Cid M.C, Cervera R. Protocolo diagnóstico de la afectación neurológica central en conectivopatías y vasculitis. Medicine 2009; 10: 2091-5.

13. Moriwaki R, Noda M, Yajima M, Sharma BK, Numano F. Clinical manifestations of Takayasu arteritis in India and Japan: new classification of angiographic findings. Angiology 1997; 48: 369-79.

14. Eichhorn J, Sima D, Thiele B, Lindschau C, Turowski A, Schmidt H, et al Anti-endothelial cell antibodies in Takayasu arteritis. Circulation 1996; 94: 2396.

15. Kissina EY, Merkela PA. Diagnostic imaging in Takayasu arteritis. Curren Opinion in Rheumatology 2004; 16: 31-7.

16. Aguirre M, Restrepo C.A. Arteritis de Takayasu en una joven de 15 años. Presentación de casos. Acta Med Colomb 2005; 30: 281-4.

17. Albers GW, Caplan LR, Easton JD, Fayad PB, Mohr JP, Saver JL, et al. Transient ischemic attack - Proposal for a new definition. N Engl J Med 2002 347: 1713-6. 